\title{
Efficacy of Dual PI3K/mTOR Inhibitor Combined With Autophagy Suppression Against Laryngeal Squamous Cell Carcinoma
}

\section{Hui-ying Huang}

Fudan University Eye Ear Nose and Throat Hospital https://orcid.org/0000-0002-5587-3576

\section{Ke-Nan Li}

Fudan University Eye Ear Nose and Throat Hospital

Hui-Ching Lau

Fudan University Eye Ear Nose and Throat Hospital

\section{Chi-Yao Hsueh}

Fudan University Eye Ear Nose and Throat Hospital

\section{Ning Cong}

Fudan University Eye Ear Nose and Throat Hospital

\section{Ming Zhang ( $\nabla$ ming.zhang@fdeent.org )}

Department of Otorhinolaryngology, Eye \& ENT Hospital, Fudan University, Shanghai, China, 200031 https://orcid.org/0000-0003-2909-8446

\section{Primary research}

Keywords: laryngeal squamous cell carcinoma (LSCC), PI3K/mTOR inhibitors, autophagy

Posted Date: June 7th, 2021

DOl: https://doi.org/10.21203/rs.3.rs-569563/v1

License: (c) (1) This work is licensed under a Creative Commons Attribution 4.0 International License. Read Full License 


\section{Abstract}

Objective: We explored the antitumor effect of dual PI3K/mTOR inhibitor combined with autophagy suppression on laryngeal squamous cell carcinoma (LSCC) and its underlying mechanism.

Methods: Hep-2 and AMC-HN-8 cell lines were treated with the Akt inhibitor LY294002, mTOR inhibitor rapamycin, and dual inhibitor NVP-BEZ235 separately. The biological characteristics of in vitro proliferation, cell cycle, apoptosis, migration, invasion, and autophagy were analyzed, and the expression levels of PI3K/Akt/mTOR pathway-related proteins were also measured. The in vivo effects of NVPBEZ235 combined with inhibition of autophagy using pharmacological inhibitor was further assessed.

Results: Compared with Akt or mTOR inhibitor, NVP-BEZ235 had the most significant biological effects on LSCC cells. When combined with various autophagy inhibitors, along with siRNA against ATG7, NVPBEZ235 showed a synergic antitumor effect in LSCC through increasing cell apoptosis and death both in vitro and vivo.

Conclusion: NVP-BEZ235 exerted stronger antitumor effects on LSCC, especially when combined with the autophagy inhibitor, providing convincing experimental data for new molecular targeted therapy for LSCC.

\section{Introduction}

Laryngeal squamous cell carcinoma (LSCC) is the most common head and neck squamous cell carcinoma (HNSCC) throughout the world, and its incidence has significantly increased in the past decade $[1,2]$. Considering that the postoperative life quality and overall survival rate of patients with LSCC remain unsatisfactory, new chemotherapy strategies or targeted therapy are urgently required [3].

The mammalian target of rapamycin (mTOR) is overactivated in many malignancies and plays a crucial part in PI3K/Akt/mTOR pathway [4], one of the most frequently overactivated pathways in most human tumors, which can promote cell growth and proliferation [5, 6], and LSCC is no exception[7]. Moreover, $\mathrm{PI} 3 \mathrm{~K} / \mathrm{Akt} / \mathrm{mTOR}$ pathway seemed to be a prognostic factor and a predictor of response to radiotherapy and chemotherapy in clinical practice [8]. Since its critical influence on tumor activity, regulation of this signaling pathway has become a potential target for LSCC intervention.

Furthermore, recent studies have indicated that autophagy inhibition could enhance anticancer therapies in various malignancies [9-11], suggesting that autophagy is also emerging as a promising therapeutic target in oncotherapy. Considering that the PI3K/Akt/mTOR pathway is also involved in the regulation of autophagy, along with the possible limitation of monotherapy with PI3K or mTOR inhibitor [12], a combination of dual PI3K/mTOR inhibitor and autophagy inhibitor has become a novel research direction. 
To identify a potential therapy strategy for LSCC, we compared the effects of three PI3K/mTOR signaling pathway inhibitors (LY294002, rapamycin, NVP-BEZ235) against LSCC cells in vitro, and the synergistic effect of the combined application of dual PI3K/mTOR inhibitor and autophagy inhibitor was evaluated in vivo.

\section{Materials And Methods 2.1. Cell lines}

Two LSCC cell lines, AMC-HN-8 and Hep-2, were acquired from the Stem Cell Bank of the Chinese Academy of Science. Both cells were cultured in RPMI-1640 medium (HyClone, Logan, UT) supplemented with $10 \%$ fetal bovine serum (FBS; Gibco, Grand Island, NY), 1\% penicillin-streptomycin (Genom Biotechnology, China), and maintained in $5 \% \mathrm{CO}_{2}$ at $37^{\circ} \mathrm{C}$.

\subsection{Reagents}

Antibodies against caspase 8, caspase 3, caspase 9, cyclin-D1, cyclin-E2, phospho-Akt (Ser473), Akt, phospho-P70S6K (Thr389), P70S6K, phospho-4E-BP1, 4E-BP1, phospho-S6RP, S6RP, LC3-II, LC3-I, and GAPDH (glyceraldehyde-3-phosphate dehydrogenase) were purchased from Sigma, USA. PI3K/mTOR inhibitors LY294002, rapamycin, NVP-BEZ235, and autophagy inhibitors 3-MA and CQ were purchased from Selleck, USA.

\subsection{MTT detection for the calculation of drug IC50}

The cell lines were prepared and then treated with different concentrations of $\operatorname{LY} 294002(1,5,10,20,40$, $80,160 \mu \mathrm{M})$, rapamycin $(1,5,10,20,40,80,160 \mu \mathrm{M})$, and NVP-BEZ235 $(0.1,0.5,1,2,4,8,16 \mu \mathrm{M})$ for $72 \mathrm{~h}$. DMSO served as the control group. Twenty microliters of MTT $(5 \mathrm{mg} / \mathrm{ml})$ was added to each well, and all plates were further incubated for $4 \mathrm{~h}$. Later, $150 \mu \mathrm{l}$ of DMSO was added, and the OD490 values were measured. The inhibition rate was selected as the $y$-coordinate and the drug concentration $(\operatorname{InX})$ as the $x$ coordinate. The fitting curve formula $Y=A * \ln X+B$ was used to calculate the semi-inhibition rate of cells, IC50 $=\exp [(0.5-b) / a]$. All assays were performed in six replicates, and each experiment was repeated three times.

\subsection{Western blotting}

Hep-2 cells were treated with or without LY294002 $(7.5 \mu \mathrm{M})$, rapamycin $(15 \mathrm{nM})$, and NVP-BEZ235 (1.5 $\mu \mathrm{M})$, while AMC-HN-8 cells were treated with or without LY294002 $(6 \mu \mathrm{M})$, rapamycin $(15 \mathrm{nM})$, and NVPBEZ235 $(0.75 \mu \mathrm{M})$. Whole cell protein lysates were obtained $72 \mathrm{~h}$ later using RIPA lysis buffer solution (Beyotime, China). Equivalent quantities of cell lysate protein $(25 \mu \mathrm{g})$ were exposed to SDS-PAGE and transferred to a PVDF membrane (Millipore, USA), which was blocked (with 5\% nonfat milk) and incubated with antibodies described above at $4^{\circ} \mathrm{C}$ overnight. The protein bands were visualized by using an enhanced chemiluminescent substrate (Millipore, USA). ImageJ software was used to analyze relative protein levels and normalize the internal reference protein GAPDH. 


\subsection{Apoptosis assay}

An Annexin V-FITC/PI Detection Kit (Invitrogen, USA) was used to examine cell apoptosis according to the manufacturer's instructions. After treatment, the LSCC cells were collected and stained with Annexin VFITC and PI. After 30 min of incubation in the dark, the cell apoptosis ratio was detected using FCM (Becton Dickinson, USA).

The TUNEL kit (Roche, Switzerland) was used to detect apoptotic cells in tumors according to the manufacturer's instructions. The proportion of TUNEL-positive cells showing a brown color on each section was counted to determine the occurrence of apoptosis.

\subsection{Cell cycle assay}

The cell cycle was examined using the propidium iodide (PI) technique. After treatment with LY294002, rapamycin, or NVP-BEZ235 $72 \mathrm{~h}$, the LSCC cells were starved for $24 \mathrm{~h}$ in RPMI-1640 without serum. Single cell suspensions were then fixed in $75 \%$ ethanol overnight at $-20^{\circ} \mathrm{C}$, washed twice with PBS, and incubated with $\mathrm{PI} / \mathrm{RNase}$ staining buffer (BD Biosciences). Samples were tested on a flow cytometer (BD Biosciences) and analyzed with FlowJo software (FlowJo LLC).

\subsection{Invasion and migration assay with a transwell chamber}

The invasive and migratory capabilities of Hep-2 and AMC-HN-8 cells following treatment with the three inhibitors were evaluated by transwell assay. For invasion, $100 \mu$ l Matrigel (Becton Dickinson, USA) was added to the bottom of each upper chamber in advance and dried for $4 \mathrm{~h}$ at $37^{\circ} \mathrm{C}$ to a gelatinous form. Then, for both experiments, $5^{\star} 10^{5} / \mathrm{ml}$ cells were placed in the upper chambers with LY294002, rapamycin, and NVP-BEZ235 incubated for $24 \mathrm{~h}$ at $37^{\circ} \mathrm{C}$. The lower chambers were filled with a culture medium with $600 \mu \mathrm{l}$ of $20 \%$ fetal bovine serum (FBS). Cells in the chambers were later fixed with $4 \%$ paraformaldehyde and stained with $0.1 \%$ crystal violet (Beyotime, China). Five random fields were selected to count, and the results were averaged and plotted.

\subsection{ATG7 siRNA Interference}

We transfected cells with siRNA oligos against ATG7 with the purpose of genetically inhibit autophagy. The sequences of ATG7 were as follows:

Atg7-1,5'-GATCCGGTGCTGGTTTCCTTGCTTAACTCGAGTTAAGCAAGGAAACCAGCACCTTTTTTG-3' and antisense, 5'-AATTCAAAAAGGTGCTGGTTTCCTTGCTTAACTCGAGTTAAGCAAGGAAACCAGCACCG-3'; Atg7-2: 5'-GATCCGCAGCCTCTCTATGAGTTTGACTCGAGTCAAACTCATAGAGAGGCTGCTTTTTTG-3' and antisense, 5'-AATTCAAAAAGCAGCCTCTCTATGAGTTTGACTCGAGTCAAACTCATAGAGAGGCTGCG-3'.

A total of $1 \times \mathrm{b} 10^{5}$ AMC-HN-8 and Hep-2 cells were seeded in 6-well plates overnight before transfection. Cells were transfected with siRNA oligonucleotides at $4 \mu \mathrm{g} / 250 \mu \mathrm{l}$ medium with $10 \mu \mathrm{l}$ Lipofectamine 2000 (Invitrogen, USA) each well according to the manufacturer's instructions.

\subsection{Xenograft nude mice of LSCC}


All male 6- to 8-week-old BALB/c nude mice (SLAC Laboratory Animal Co., Ltd, Shanghai) were inoculated with $6 \times 10^{6}$ Hep-2 cells before being randomly divided into four groups ( $n=6$ each) as follows: (1) control group; (2) NVP-BEZ235 group, oral administration of NVP-BEZ235 (30 mg/kg/d); (3) CQ group, intraperitoneal (i.p.) administration of CQ $(50 \mathrm{mg} / \mathrm{kg} / \mathrm{d})$; (4) NVP-BEZ235 + CQ group, rats were treated with NVP-BEZ235 (30 mg/kg/d, p.o.) and CQ (50 mg/kg/d, i.p.) simultaneously. The tumor sizes were measured twice a week with calipers. All surviving rats were sacrificed 28 days later to collect tumors for further detection. Tumor size and tumor cell apoptosis of each group were analyzed.

\subsection{Statistical analysis}

Data analysis was accomplished using SPSS 22.0 software. Differences between groups were assessed by the Student's t-test (two-tailed), and the results are shown as means \pm SEM. $P<0.05$ was considered statistically significant.

\section{Results}

\subsection{Dual PI3K/mTOR inhibitor NVP-BEZ235 leads to cell cycle arrest and apoptosis in LSCC cell lines}

To clarify the role of PI3K/Akt/mTOR pathway in LSCC, Hep-2 and AMC-HN-8 cell lines were treated with PI3K inhibitor LY294002, mTOR inhibitor rapamycin, and dual PI3K/mTOR inhibitor NVP-BEZ235 separately. Cell apoptosis and the cell cycle were then assessed. NVP-BEZ235 elicited significant blockage at G2/M phase, and the protein levels of cyclin D1 and cyclin E2 also decreased in both cell lines than LY294002 and rapamycin (Fig. 1), which suggested its better effect on the cell cycle as a dual $\mathrm{PI} 3 \mathrm{~K} / \mathrm{mTOR}$ inhibitor. Treatment with all three inhibitors resulted in significant apoptosis in Hep-2 and AMC-HN-8, especially with NVP-BEZ235 (Fig. 2A, 2B), whereas the protein level of caspases 3, 8, and 9 increased after inhibitor treatment (Fig. 2C).

\subsection{NVP-BEZ235 shows more potent and dual blocking effects for MTORC1 and mTORC2}

We next tested the activation of AKT, p70S6K, 4EBP1, and S6RP in the two cell lines after the treatment with the inhibitors. NVP-BEZ235 modulated not only mTORC1 targets p-70S6K, p-4EBP1 and p-S6RP, but also mTORC2 targets p-Akt (Fig. 3). To evaluate antitumor effects, we further manipulated the migration and invasion test by transwell chamber assay in Hep-2 and AMC-HN-8 treated with Ly294002, rapamycin, NVP-BEZ235, or DMSO (as control). All three inhibitors reduced cell migration and invasion in vitro (Fig. 4A, 4B, 4C, 4D); however, NVP-BEZ235 elicited the most significant effect.

Considering the above assessment, NVP-BEZ235 had a better block effect on PI3K/Akt/mTOR pathway as a dual PI3K/mTOR inhibitor on both LSCC cell lines than LY294002 and rapamycin. 


\subsection{Synergic antitumor effect of combination NEP-BEZ235 with autophagy inhibition in vitro}

As NVP-BEZ235 can regulate cell autophagy in many tumors $[13,14]$, we investigated the regulatory effects of all three inhibitors on autophagy in LSCC cell lines. Autophagy control was observed using a confocal laser scanning microscope after acridine orange staining (Supplemental Fig. 1) and verified by LC3, a protein embedded in autophagosomes that became lipids during active autophagy. LC3II/LC3I ratio was obtained using western blotting analysis (Fig. 5D. E). We observed that all three inhibitors of $\mathrm{PI3K} / \mathrm{mTOR}$ pathway induced autophagy, indicated by the rise of LC3II/LC3I ratio, with NVP-BEZ235 potentiating the ratio more effectively.

Furthermore, we tested the combination of NVP-BEZ235 with autophagy inhibitors, 3-methyladenine (3$M A)$, chloroquine (CQ) and ATG7-SiRNA. 3-MA inhibits the initiation of autophagy, CQ inhibits the fusion of autophagosomes and lysosomes; and ATG7-SiRNA, is a genetic silencing of Atg7, which inhibits the early phase of autophagy. The results showed that cell death was significantly increased when NVPBEZ235 was combined with autophagy inhibitors (Fig. 5A. B. C), although LC3II/LC3I ratio and the expression level of caspase 3 were not significantly changed by the combination. This result strongly indicates that the combination of NVP-BEZ235 and autophagy inhibitors has a synergic antitumor effect through increasing LSCC cell apoptosis and death.

\subsection{Synergic antitumor effect of combination NEP-BEZ235 with autophagy inhibition in vivo}

We then tested the antitumor efficacy of the combination of NVP-BEZ235 with CQ in vivo. BALB/c mice bearing Hep-2 xenograft tumors were treated with NVP-BEZ235, CQ, NVP-BEZ235 + CQ, and physiological saline solution as a control for 4 weeks after tumor cell injection. First, we tested the drug toxicity of all groups by evaluating the changes in animal weight, behavior, and appearance. The tumor size was then measured weekly before the animals were sacrificed. The final tumor masses were dissected after the last measurement. Combination of NVP-BEZ235 and CQ demonstrated synergistic effect against autophagy, indicated by reduced tumor size and final tumor weight (Fig. 6). The interaction effect was elicited by the interaction index $(p<0.0001)$. Based on TUNEL assay of fragmented DNA of apoptotic cells in final tumor masses, the most apoptotic cells were found in the group treated with NVP-BEZ235 and CQ.

\section{Discussion}

In this study, the dual PI3K/mTOR inhibitor NVP-BEZ235 revealed a better effect on the cell cycle, cell apoptosis, migration, and invasion in LSCC. The expression changes in downstream molecules of $\mathrm{PI} 3 \mathrm{~K} / \mathrm{Akt} / \mathrm{mTOR}$ signaling pathway further explained its underlying mechanism. We demonstrated that the combination of NVP-BEZ235 and autophagy inhibitors has a synergic antitumor effect by promoting cell apoptosis and death both in vitro and vivo. 
Since PI3K/Akt/mTOR signaling pathway plays a crucial role in tumorigenesis by strikingly promoting cell growth and proliferation $[15,16]$, inhibition of this pathway is also postulated as a therapeutic option in LSCC[17]. The mTOR protein serves as an integral part in this signaling complex, which is involved in two major complexes: mTOR complex1 (mTORC1) and mTOR complex2 (mTORC2)[18]. Although discovered much later than mTORC1, mTORC2 is considered essential for transformation and viability of cancer cells while it appears more important in tumor cells, indicating mTORC2 is emerging as a promising candidate target in oncotherapy [19]. Studies have proved that mTORC2 can directly regulate Akt phosphorylation via activating a critical regulatory site required for maximal Akt kinase activity, which enables Akt to become a crucial downstream protein of the mTORC2 signal pathway [20,21]. Due to multiple resistance mechanisms, such as the feedback activation of the PI3K/Akt signaling network and the insensitivity of the mTORC1 complex, inhibition of the MTORC1 pathway has met with limited success in many studies [22-25], which is also verified in our experiment. Compared with Akt inhibitor LY294002 and mTORC1 inhibitor rapamycin [26], dual PI3K/mTOR inhibitor NVP-BEZ235 showed better anticancer effects in arresting the cell cycle, promoting cell apoptosis, and inhibiting cell invasion and metastasis by suppressing mTORC1 and mTORC2 at the same time. Changes in downstream proteins, such as p-Akt, p-4E-BP1, p-S6RP, and p-p70S6K, also supported the remarkable anticancer effect of NVPBEZ235 and its corresponding mechanism in two LSCC cell lines.

However, PI3K/mTOR inhibitors still have a moderate effect as single agents in clinical trials, which may be related to their induction of cell autophagy $[27,28]$. Through degradation of damaged proteins and organelles, autophagy can protect tumor cells from stressful conditions, thus mediating resistance to anticancer therapies such as radiation, chemotherapy, and targeted treatments[29-32]. In this study, an increase of LC3II/I ratio was found in both LSCC cell lines treated with PI3K or mTOR inhibitors, among which the dual inhibitor NVP-BEZ235 is the most significant, supporting its induction of cell autophagy. Based on accumulating evidence that inhibition of autophagy can enhance the efficiency of antitumor drugs [33-35], we combined NVP-BEZ235 with autophagy inhibitors for further verification.

Surprisingly, the combination of NVP-BEZ235 with two autophagy inhibitors (3-MA, CQ) showed a synergetic antitumor effect in both cell lines. LSCC cells that inhibited autophagy in the early phase by silencing Atg7 also had increased cell apoptosis and death, further indicating that inhibition of autophagy can effectively improve the antitumor effect of PI3K/Akt inhibitors. We then combined NVPBEZ235 with the less toxic autophagy inhibitor CQ to test their antitumor efficacy in vivo. The remarkably increased proportion of apoptotic cells in the tumor, along with the obviously decreased tumor size and tumor weight, were all consistent with those of our previous cell experiments.

In summary, we have proved the remarkable antitumor effect of dual PI3K/Akt inhibitor NVP-BEZ235 compared to other PI3K or Akt inhibitors in LSCC cells, which may be achieved by inhibiting mTORC1 and mTORC2 simultaneously. Combined treatment with $\mathrm{CQ}$, which could inhibit autophagy induced by NVPBEZ235, further promoted tumor cell apoptosis and death both in vitro and vivo, indicating their potential synergic antitumor effect in LSCC. Based on the current trend of combination therapy in cancer treatment [36], our experiments have solved the limitations of PI3K/Akt inhibitors to some extent, suggesting that 
combined application of autophagy inhibitors could be an effective way to overcome resistance of LSCC to $\mathrm{PI3K} /$ Akt inhibitors, while providing a promising therapy with the combined application of targeted drugs in LSCC.

\section{Declarations}

\section{Ethics approval and consent to participate:}

This study did not involve the acquisition of HSCC tissue samples. The animal experiments were approved by the Animal Center, EENT Hospital, Fudan University

\section{Consent for publication:}

All the authors listed have approved the manuscript that is enclosed.

\section{Availability of data and materials:}

All the source data supporting the findings of this study are available from the corresponding author upon reasonable request.

\section{Competing interests:}

The authors declare no conflict of interest.

\section{Funding:}

The present study was supported by grants from Natural Science Foundation of Shanghai [No. 17ZR1404700].

\section{Authors' contributions:}

Hui-Ying Huang: Conceptualization, Formal analysis, Investigation, Methodology, Project administration, Validation, Writing - original draft, Writing - review \& editing. Ke-Nan Li: Formal analysis, Investigation, Resources, Validation, Writing - original draft. Hui-Ching Lau: Formal analysis, Investigation, Resources, Validation. Chi-Yao Hsueh: Project administration, Resources. Ning Cong: Conceptualization, Project administration, Resources, Supervision, Writing - review \& editing. Ming Zhang: Conceptualization, Funding acquisition, Resources, Supervision, Writing - review \& editing 


\section{Acknowledgements:}

The authors declare no conflict of interest.

\section{References}

1. Torre, L.A., et al., Global cancer statistics, 2012. CA Cancer J Clin, 2015. 65(2): p. 87-108.

2. Parkin, D.M., et al., Global cancer statistics, 2002. CA Cancer J Clin, 2005. 55(2): p. 74-108.

3. Nwizu, T. and D. Adelstein, Pharmacotherapy of head and neck cancer. Expert Opin Pharmacother, 2015. 16(16): p. 2409-22.

4. Zhou, C., et al., SLFN11 inhibits hepatocellular carcinoma tumorigenesis and metastasis by targeting RPS4X via mTOR pathway. Theranostics, 2020. 10(10): p. 4627-4643.

5. Lui, V.W., et al., Frequent mutation of the PI3K pathway in head and neck cancer defines predictive biomarkers. Cancer Discov, 2013. 3(7): p. 761-9.

6. Garcia-Carracedo, D., et al., Impact of PI3K/AKT/mTOR pathway activation on the prognosis of patients with head and neck squamous cell carcinomas. Oncotarget, 2016. 7(20): p. 29780-93.

7. Yun, M.R., et al., ERK-dependent IL-6 autocrine signaling mediates adaptive resistance to pan-PI3K inhibitor BKM120 in head and neck squamous cell carcinoma. Oncogene, 2018. 37(3): p. 377-388.

8. Martini, M., et al., PI3K/AKT signaling pathway and cancer: an updated review. Ann Med, 2014. 46(6): p. 372-83.

9. Nunez-Olvera, S.I., et al., Autophagy Machinery as a Promising Therapeutic Target in Endometrial Cancer. Front Oncol, 2019. 9: p. 1326.

10. Fan, Q.W., et al., Akt and autophagy cooperate to promote survival of drug-resistant glioma. Sci Signal, 2010. 3(147): p. ra81.

11. Rubinsztein, D.C., et al., Potential therapeutic applications of autophagy. Nat Rev Drug Discov, 2007. 6(4): p. 304-12.

12. Salazar, R., et al., Phase II Study of BEZ235 versus Everolimus in Patients with Mammalian Target of Rapamycin Inhibitor-Naive Advanced Pancreatic Neuroendocrine Tumors. Oncologist, 2018. 23(7): p. 766-e90.

13. Akkoc, Y., et al., Inhibition of PI3K signaling triggered apoptotic potential of curcumin which is hindered by Bcl-2 through activation of autophagy in MCF-7 cells. Biomed Pharmacother, 2015. 71: p. 161-71.

14. Seitz, C., et al., The dual PI3K/mTOR inhibitor NVP-BEZ235 and chloroquine synergize to trigger apoptosis via mitochondrial-lysosomal cross-talk. International Journal of Cancer, 2013. 132(11): p. 2682-2693.

15. Zoncu, R., A. Efeyan, and D.M. Sabatini, mTOR: from growth signal integration to cancer, diabetes and ageing. Nat Rev Mol Cell Biol, 2011. 12(1): p. 21-35. 
16. Xu, F., et al., Roles of the PI3K/AKT/mTOR signalling pathways in neurodegenerative diseases and tumours. Cell Biosci, 2020. 10: p. 54.

17. Marquard, F.E. and M. Jücker, PI3K/AKT/mTOR signaling as a molecular target in head and neck cancer. Biochemical pharmacology, 2020. 172: p. 113729.

18. Laplante, M. and D.M. Sabatini, mTOR signaling in growth control and disease. Cell, 2012. 149(2): p. 274-93.

19. Sparks, C.A. and D.A. Guertin, Targeting mTOR: prospects for mTOR complex 2 inhibitors in cancer therapy. Oncogene, 2010. 29(26): p. 3733-44.

20. Beauchamp, E.M. and L.C. Platanias, The evolution of the TOR pathway and its role in cancer. Oncogene, 2013. 32(34): p. 3923-32.

21. Sarbassov, D.D., et al., Phosphorylation and regulation of Akt/PKB by the rictor-mTOR complex. Science, 2005. 307(5712): p. 1098-101.

22. Menon, S. and B.D. Manning, Common corruption of the mTOR signaling network in human tumors. Oncogene, 2008. 27 Suppl 2: p. S43-51.

23. O'Reilly, K.E., et al., mTOR inhibition induces upstream receptor tyrosine kinase signaling and activates Akt. Cancer Res, 2006. 66(3): p. 1500-8.

24. Sun, S.Y., et al., Activation of Akt and elF4E survival pathways by rapamycin-mediated mammalian target of rapamycin inhibition. Cancer Res, 2005. 65(16): p. 7052-8.

25. Tamburini, J., et al., Mammalian target of rapamycin (mTOR) inhibition activates phosphatidylinositol 3-kinase/Akt by up-regulating insulin-like growth factor-1 receptor signaling in acute myeloid leukemia: rationale for therapeutic inhibition of both pathways. Blood, 2008. 111(1): $p$. 379-82.

26. Brown, E.J., et al., A mammalian protein targeted by G1-arresting rapamycin-receptor complex. Nature, 1994. 369(6483): p. 756-8.

27. Xin, P., et al., Efficacy of the dual PI3K and mTOR inhibitor NVP-BEZ235 in combination with imatinib mesylate against chronic myelogenous leukemia cell lines. Drug Des Devel Ther, 2017. 11: p. 11151126.

28. Wu, N., et al., The anti-tumor effects of dual PI3K/mTOR inhibitor BEZ235 and histone deacetylase inhibitor Trichostatin A on inducing autophagy in esophageal squamous cell carcinoma. J Cancer, 2018. 9(6): p. 987-997.

29. Cheng, Y., et al., Therapeutic targeting of autophagy in disease: biology and pharmacology. Pharmacol Rev, 2013. 65(4): p. 1162-97.

30. Levy, J.M.M., C.G. Towers, and A. Thorburn, Targeting autophagy in cancer. Nat Rev Cancer, 2017. 17(9): p. 528-542.

31. He, J., et al., Downregulation of ATG14 by EGR1-MIR152 sensitizes ovarian cancer cells to cisplatininduced apoptosis by inhibiting cyto-protective autophagy. Autophagy, 2015. 11(2): p. 373-84. 
32. $\mathrm{He}, \mathrm{H}$., et al., Upregulation of KCNQ10T1 promotes resistance to stereotactic body radiotherapy in lung adenocarcinoma by inducing ATG5/ATG12-mediated autophagy via miR-372-3p. Cell Death Dis, 2020. 11(10): p. 883.

33. Phan, T., et al., Inhibition of Autophagy Amplifies Baicalein-Induced Apoptosis in Human Colorectal Cancer. Mol Ther Oncolytics, 2020. 19: p. 1-7.

34. Corallo, D., et al., Autophagic flux inhibition enhances cytotoxicity of the receptor tyrosine kinase inhibitor ponatinib. J Exp Clin Cancer Res, 2020. 39(1): p. 195.

35. Zou, Y., et al., The autophagy inhibitor chloroquine overcomes the innate resistance of wild-type EGFR non-small-cell lung cancer cells to erlotinib. J Thorac Oncol, 2013. 8(6): p. 693-702.

36. Moghaddam, S.V., et al., Lysine-embedded cellulose-based nanosystem for efficient dual-delivery of chemotherapeutics in combination cancer therapy. Carbohydr Polym, 2020. 250: p. 116861.

\section{Figures}

A

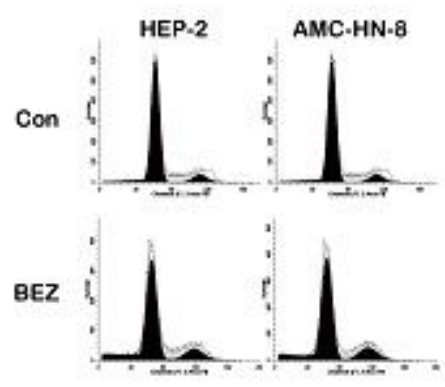

8

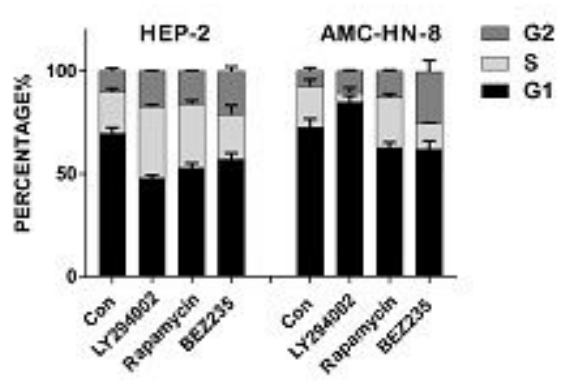

C

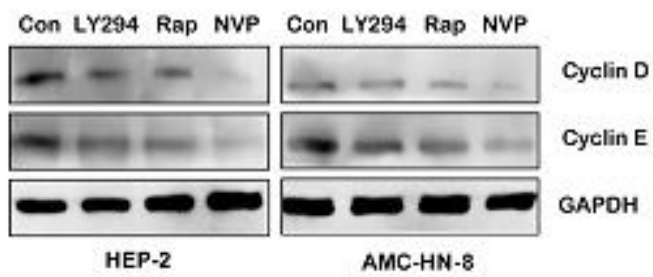

Figure 1

Cell cycle is affected by inhibitors of PI3K/ mTOR pathway. (A) Flow cytometric (FCM) analysis of cultured Hep-2 and AMC-HN-8 cells. (B) Cell cycle analysis of the Hep-2 and AMC-HN-8 cell lines. Cells in G2/M phase were remarkably increased by NVP-BEZ235 in both cell lines. Columns, means of duplicate measurements; bars, \pm SD. (C) Western blotting analysis of the expression levels of cyclin D1 and cyclin 
E2 in two cell lines treated with three different PI3K/ mTOR inhibitors. Cyclin D1 and cyclin E2 decreased significantly after treatment with NVP-BEZ235.

A
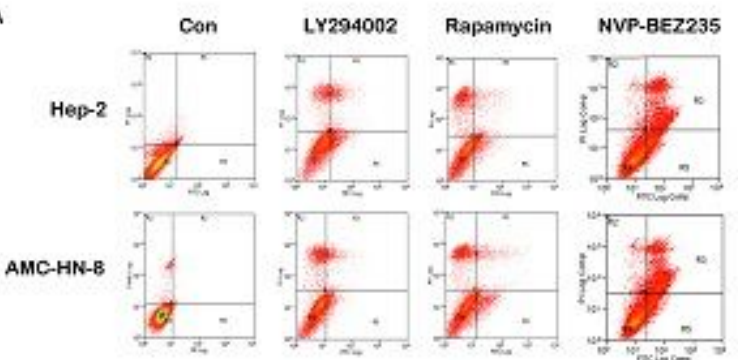

B

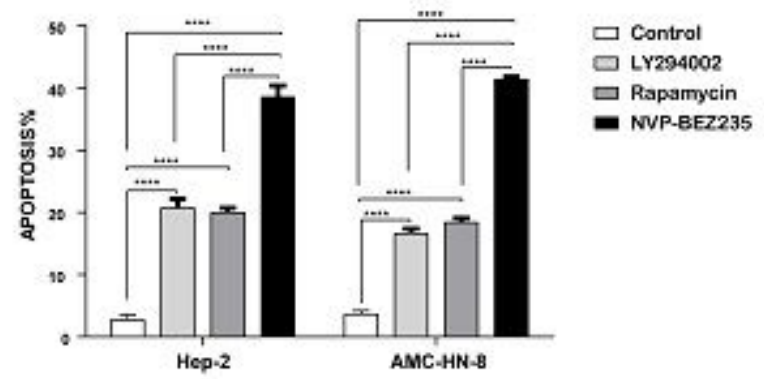

c

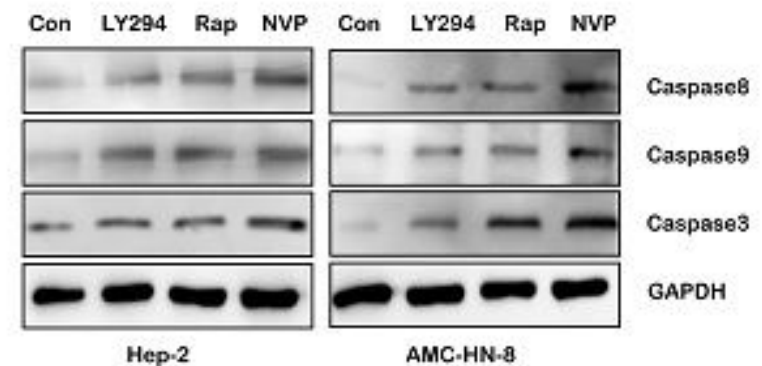

Figure 2

Cell apoptosis is increased by inhibitors of PI3K/mTOR pathway. (A) Flow cytometry (FCM) analysis of the cell apoptosis of cultured Hep-2 and AMC-HN-8 cell lines. (B) Cell apoptosis was increased by all three inhibitors in both cell lines ( $p$ <.0001). The apoptosis increase was similar with Ly294002 and rapamycin, but was more pronounce with NVP-BEZ235 ( $<<0.0001)$. Columns, means of duplicate measurements; bars, \pm SD. (C) Caspases 8, 9, and 3 expression in the Hep-2 and AMC-HN-8 cell lines increased after NVP-BEZ235 treatment. 


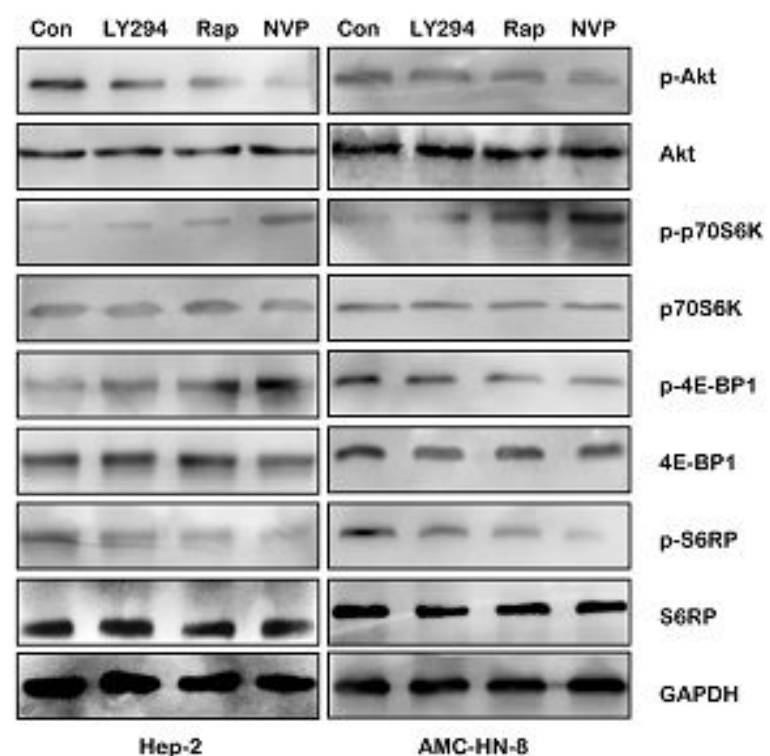

\section{Figure 3}

Protein expression and phosphorylation of members of the PI3K/Akt/mTOR pathway of Hep-2 and AMCHN-8 cell lines, assessed by western blotting.

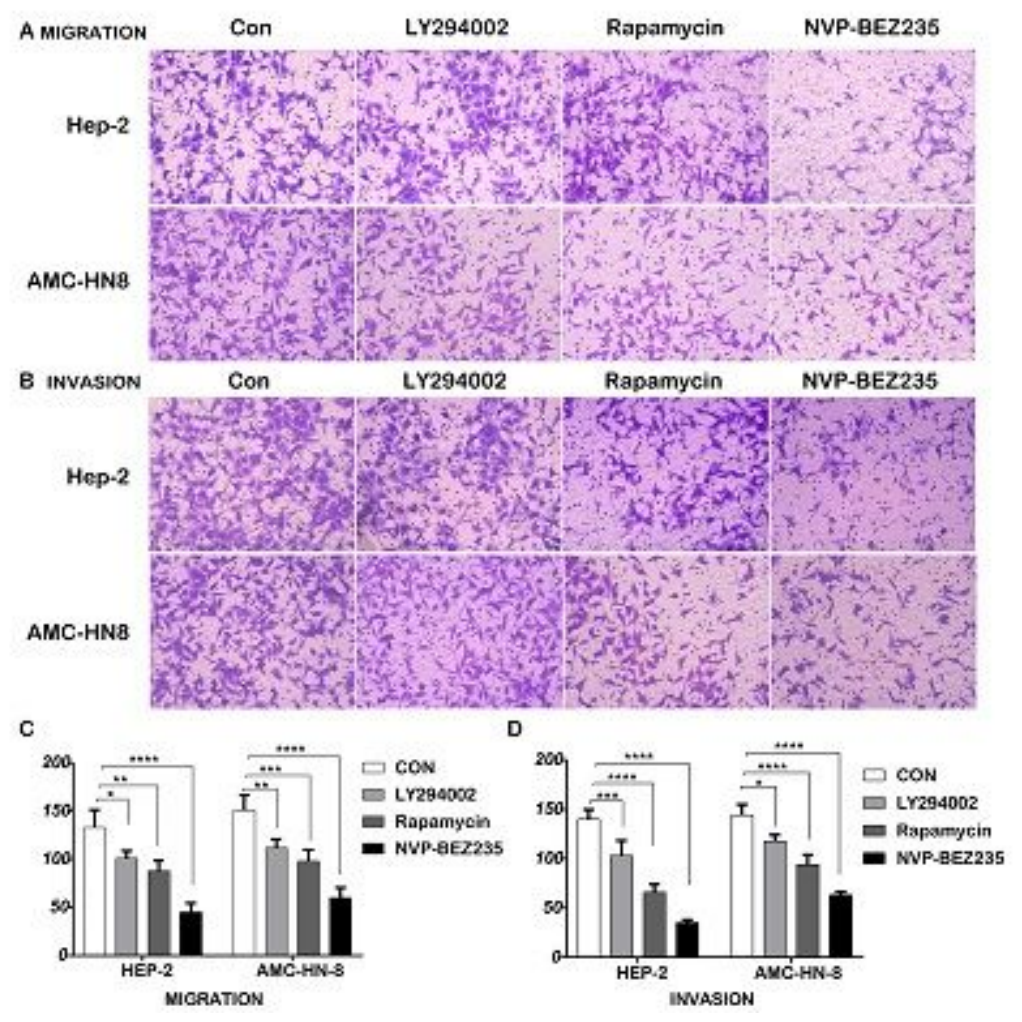

\section{Figure 4}

Cell migration and invasion is modulated by PI3K/ mTOR inhibitors. (A), (B) Migration and invasion affected by LY294002, rapamycin, and NVP-BEZ235 in Hep-2 and AMC-HN-8 cell lines were separately tested by a trans-well chamber assay. (C), (D) Cell quantification showed a significant decrease in 
migration and invasion by all three inhibitors in both cell lines, and NVP-BEZ235 showed a stronger effect.

A
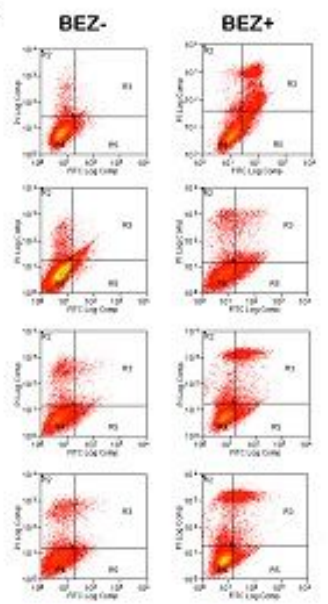

HEP-2
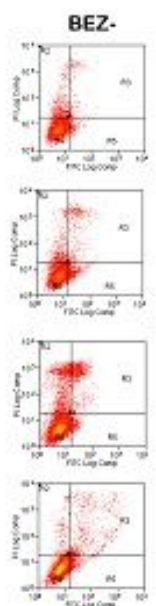

AMC-HN-B
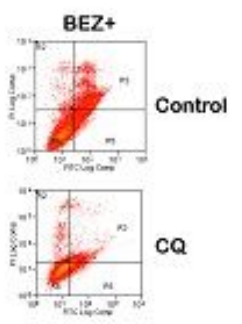

Q
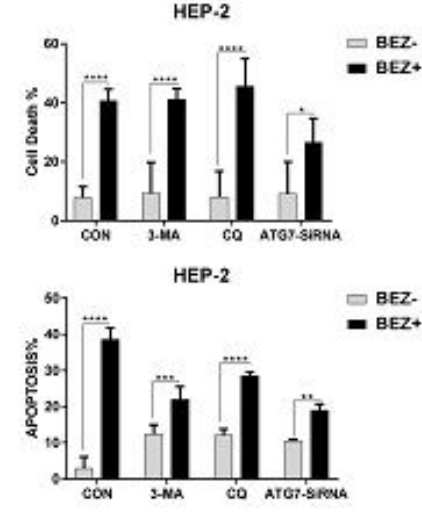

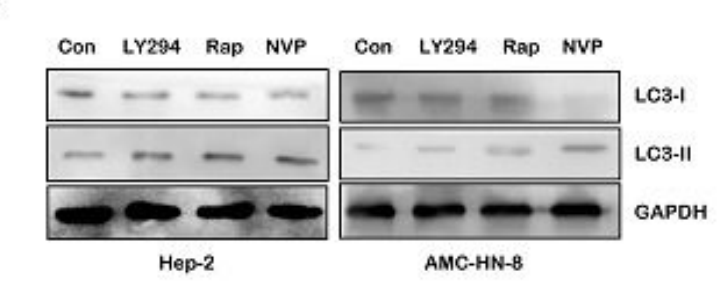

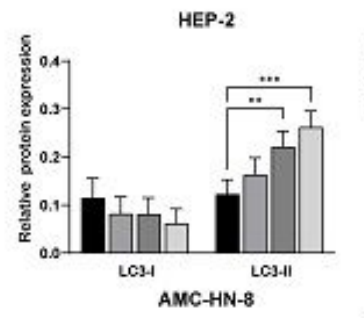

HEP-2

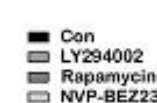

믈 RVpamycin

NVP-BEZ235
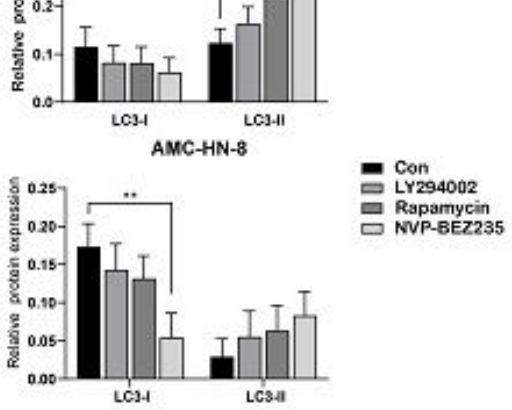

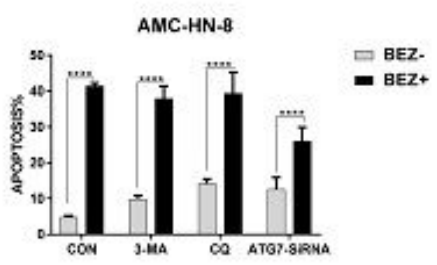

AMC-HN-8
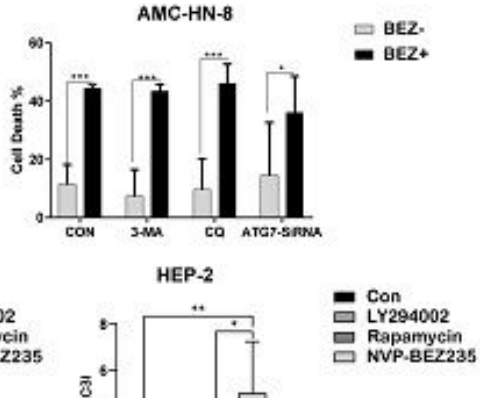
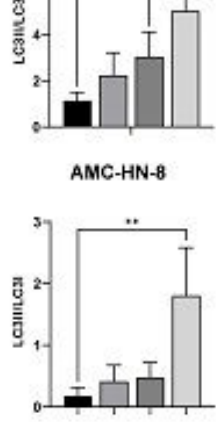

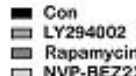

口 NVP-BEZ235

\section{Figure 5}

Cell autophagy and of PI3K/Akt/mTOR pathway inhibition. (A) Flow cytometric (FCM) analyses of the cell apoptosis of cultured Hep-2 cells or AMC-HN-8 cells. (B) Cell apoptosis increased in both cell lines treated with a combination of NVP-BEZ235 and autophagy inhibitors. Columns, means of duplicate measurements; bars \pm SD. (C) Cell death increased in both cell lines treated with a combination of NVPBEZ235 and autophagy inhibitors. (D) Western blotting analysis of levels of LC3II and LC3I expression in two cell lines treated with three PI3K inhibitors. (E) Western blotting analysis showed LC3II/I ratio decreased significantly following treatment of both cell lines with Ly294002, rapamycin, and NVPBEZ235. 

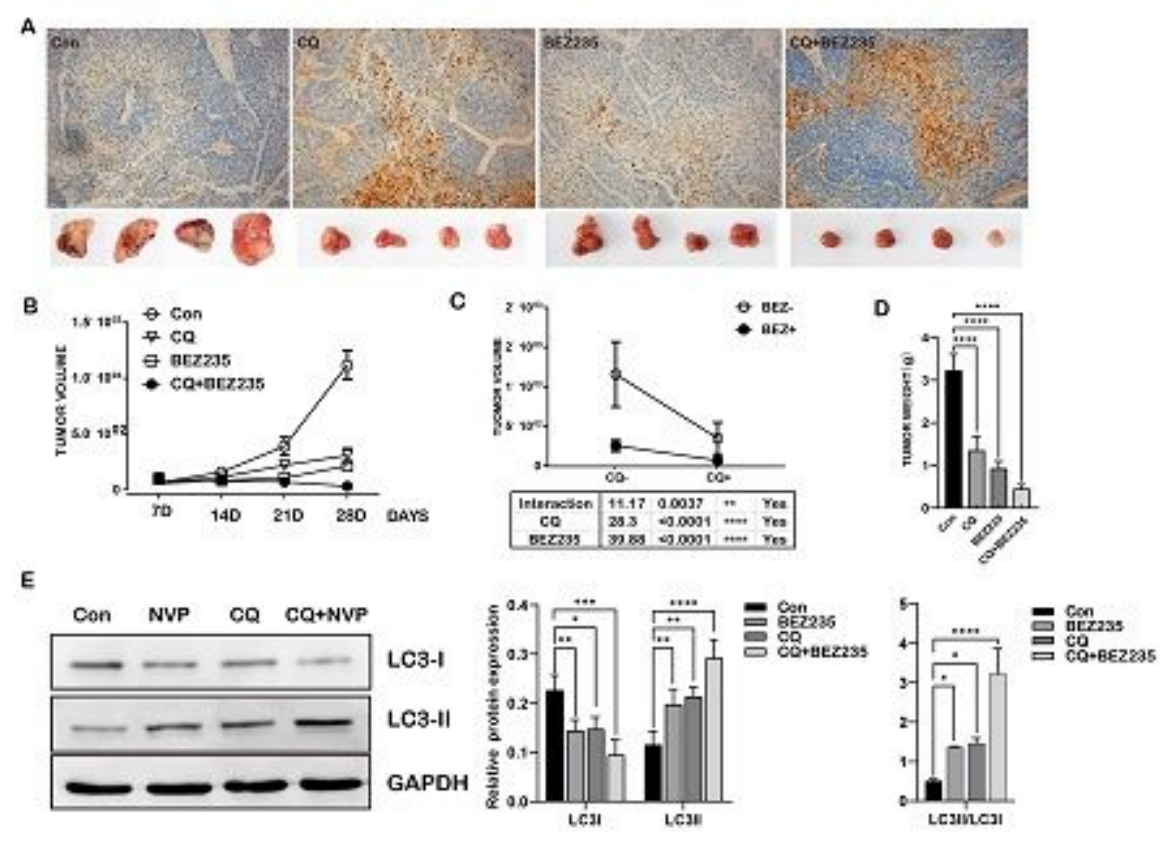

\section{Figure 6}

Anti-tumor efficacy of the combination of NVP-BEZ235 with autophagy inhibitor in vivo. (A) Tumors obtained from mice and their immunohistochemical results. (B) Tumor volumes increased with days, and their analysis results. Points, mean of four replicate determinations; bars \pm SD. (C), (D) Differences in tumor weights between the four groups. Points and columns, mean of four replicate determinations; bars \pm SD. (E) Western blotting testing for LC3II and LC3I expression of tumors from the four groups and the corresponding analysis.

\section{Supplementary Files}

This is a list of supplementary files associated with this preprint. Click to download.

- Fig.S1.tif

- Fig.S2.tif 